\title{
Rotational Brownian Motion of an Asymmetric Top
}

\author{
G.W. FORD \\ Dept. of Physics. The University of Michigan, Ann Arbor, Michigan 48109, U.S.A.
}

Some time ago J.T. Lewis, J. McConnell and I published a paper with this same title in which we showed how methods of stochastic differential equations could be used to discuss the motion of an asymmetric top driven by white noise random torques [1]. Since the detailed results, including the application to the calculation of complex polarizabilities of asymmetric top molecules and the correlation times associated with the dipolar broadening of nuclear magnetic resonance lines, are given in that paper, I will not give them here and will instead emphasize the general methods. In part these methods are those described in a set of unpublished "Notes on Stochastic Differential Equations" which I wrote in June 1974, inspired by a visit and talk at the University of Michigan by N.G. van Kampen. In his talk van Kampen described the methods based on the cumulant expansion of the time-ordered exponential which he and, independently, R.F. Fox had developed for handling multiplicative stochastic equations [2,3]. I realized that the same results could be obtained by an extension of the Method of Averaging of Krylov and Bogoliubov [4,5], a well known method of nonlinear mechanics, and that is what is described in the above mentioned notes. The averaging method applies to nonlinear as well as to linear stochastic differential equations and the application to rotational Brownian motion is in part an illustration of this advantage.

The fundamental equations of motion are the Euler-Langevin equations

$$
\mathrm{d} \boldsymbol{L} / \mathrm{d} t+\boldsymbol{\omega} \times \boldsymbol{L}+\mathbf{F} \cdot \boldsymbol{\omega}=\boldsymbol{N}(t),
$$

where $\boldsymbol{L}$ is the angular momentum and $\boldsymbol{\omega}$ the angular velocity, related by the moment of inertia tensor I,

$$
\boldsymbol{L}=\mathbf{I} \cdot \boldsymbol{\omega}
$$

This is just the familiar Euler equation in a body-fixed coordinate frame with an added frictional torque $\mathbf{F} \cdot \boldsymbol{\omega}$ and a random torque $\boldsymbol{N}(t)[6]$. The friction tensor $\mathbf{F}$ is assumed to be constant and to commute with the moment of inertia tensor, so it may be written

$$
\mathbf{F}=\mathbf{I} \cdot \mathbf{B}=\mathbf{B} \cdot \mathbf{I}
$$

where $\mathbf{B}$ is the frictional decay rate tensor. The constant friction tensor implies, via the fluctuationdissipation theorem $[7,8]$, that the random torque must be Gaussian white noise with covariance

$$
\left\langle\boldsymbol{N}(t) \mathbf{N}\left(t^{\prime}\right)\right\rangle=2 k T \mathbf{F} \delta\left(t-t^{\prime}\right)
$$

We seek the stationary process $\omega(t)$, which is a solution of (1) with mean zero,

$$
\langle\omega(t)\rangle=0
$$


This can be written in the form of a perturbation series

$$
\omega(t)=\omega^{(1)}(t)+\omega^{(2)}(t)+\omega^{(3)}(t)+\cdots,
$$

where $\omega^{(n)}(t)$ is a homogeneous functional of degree $n$ in $N(t)$. Explicitly,

$$
\boldsymbol{\omega}^{(1)}(t)=\mathbf{I}^{-1} \cdot \int_{-\infty}^{t} \mathrm{~d} t^{\prime} \exp \left\{-\mathbf{B}\left(t-t^{\prime}\right)\right\} \cdot N\left(t^{\prime}\right)
$$

and

$$
\boldsymbol{\omega}^{(n)}(t)=\mathbf{I}^{-1} \cdot \int_{-\infty}^{t} \mathrm{~d} t^{\prime} \exp \left\{-\mathbf{B}\left(t-t^{\prime}\right)\right\} \cdot \prod_{j=1}^{n-1} \boldsymbol{I} \cdot \boldsymbol{\omega}^{(n-j)}\left(t^{\prime}\right) \times \boldsymbol{\omega}^{(j)}\left(t^{\prime}\right)
$$

The first order term $\omega^{(1)}(t)$ is a Gaussian process with covariance

$$
\left\langle\boldsymbol{\omega}^{(1)}(t) \boldsymbol{\omega}^{(1)}\left(t^{\prime}\right)\right\rangle=k T \mathbf{I}^{-1} \cdot \exp \left\{-\mathbf{B} \mid t-t^{\prime}\right\}
$$

This is just the covariance of the well-known Ornstein-Uhlenbeck process, the stationary solution of the Langevin equation which results when the nonlinear term in (1) is dropped. A result of the presence of this nonlinear term is that the process $\omega(t)$ is not Gaussian. In particular this means that in general correlations involving an odd number of factors $\omega(t)$ do not vanish (except for the first). Nevertheless, the expansion (6) and the expressions (7) and (8) can be used to calculate the various correlations of $\omega(t)$ to any given order. When we do this, e.g., for the autocorrelation, we see explicitly that the series (6) is an expansion in powers of the parameter

$$
(k T / I)^{1 / 2} B^{-1}
$$

where $I$ is a typical moment of inertia (eigenvalue of $I$ ) and $B$ is a typical frictional decay rate (eigenvalue of $\mathbf{B}$ ). Physically this parameter is the ratio of the mean thermal angular velocity of rotation to the rate of decay of a fluctuation of angular velocity and is small near the rotational diffusion limit, where inertial effects are small. This means that our results are useful only near that limit, what we calculate are the inertial corrections to diffusion.

The process $\omega(t)$ "drives" the rotational motion of the top. We describe this in terms of a matrix or operator $R(t)$ which describes the orientation of the body-fixed coordinate axes relative to the space fixed axes, which satisfies the kinematical equation of motion:

$$
\mathrm{d} R / \mathrm{d} t=\omega(t) \cdot \boldsymbol{\sigma} R
$$

where

$$
\omega(t) \cdot \sigma=\omega_{x}(t) \sigma_{x}+\omega_{y}(t) \sigma_{y}+\omega_{z}(t) \sigma_{z}
$$


in which the components of $\omega(t)$ are referred to the body fixed axes and $\sigma_{x}, \sigma_{y}$ and $\sigma_{z}$ are time-independent antihermetian operators, generators of infinitesimal rotations, which satisfy the commutation relation

$$
\left[\sigma_{x}, \sigma_{y}\right]=-\sigma_{z}
$$

and cyclic permutations. In the simplest case $R(t)$ is the $3 \times 3$ matrix of the direction cosines between the body-fixed and space-fixed axes and

$$
\sigma_{x}=\left[\begin{array}{rrr}
0 & 0 & 0 \\
0 & 0 & 1 \\
0 & -1 & 0
\end{array}\right], \quad \sigma_{y}=\left[\begin{array}{rrr}
0 & 0 & -1 \\
0 & 0 & 0 \\
1 & 0 & 0
\end{array}\right], \quad \sigma_{t}=\left[\begin{array}{rrr}
0 & 1 & 0 \\
-1 & 0 & 0 \\
0 & 0 & 0
\end{array}\right] .
$$

Equation (11) is of the form of an equation for a multiplicative stochastic process, multiplicative because the random driving term $\omega(t)$ is multiplicative, in contrast to the additive random torque in (1). The solution of this equation is a random process $R(t)$. The physically observed quantities are expressed in terms of the after-effect function, which is the mean $\langle R(t)\rangle$ subject to the condition that at $t=0$ the top is oriented so that the body-fixed and space-fixed axes coincide. That is

$$
R(0)=\langle R(0)\rangle=1
$$

Multiplicative stochastic equations of the form (11) have much in common with the equations of motion for parametrically driven mechanical systems, which in turn have much in common with the equations for nonlinear mechanical systems. As I indicated above, we apply a method based on the averaging methods of nonlinear mechanics to discuss our equation, which we rewrite in the form

$$
\mathrm{d} R / \mathrm{d} t=\left[\varepsilon K^{(1)}(t)+\varepsilon^{2} K^{(2)}(t)+\cdots\right] R,
$$

where

$$
\varepsilon^{n} K^{(n)}(t)=\omega^{(n)}(t) \cdot \sigma
$$

Here the small parameter $\varepsilon$ has been exhibited in order to keep track of orders; we can think of it as the parameter (10). Now for $\varepsilon$ small, the solution of (16) must consist of a slowly varying mean $\langle R(t)\rangle$ about which there will be small-amplitude fluctuations. We therefore seek a solution in the form

$$
R(t)=\left[1+\varepsilon F^{(1)}(t)+\varepsilon^{2} F^{(2)}(t)+\cdots\right]\langle R(t)\rangle,
$$

where $F^{(n)}(t)$ is a stochastic operator with mean zero

$$
\left\langle F^{(n)}(t)\right\rangle=0
$$

The mean $\langle R(t)\rangle$ must satisfy a nonstochastic differential equation of the form

$$
\mathrm{d}\langle R(t)\rangle / \mathrm{d} t=\left[\varepsilon \Omega^{(1)}(t)+\varepsilon^{2} \Omega^{(2)}(t)+\cdots\right]\langle R(t)\rangle,
$$


where $\Omega^{(n)}(t)$ is a nonstochastic operator. Inserting (18) in (16) and using (20), we then equate coefficients of equal powers of $\varepsilon$ on either side to get a sequence of equations for determining the $F$ 's and the $\Omega$ 's:

$$
\begin{aligned}
& \Omega^{(1)}+\mathrm{d} F^{(1)} / \mathrm{d} t=K^{(1)}, \\
& \Omega^{(2)}+\mathrm{d} F^{(2)} / \mathrm{d} t=K^{(2)}+K^{(1)} F^{(1)}-F^{(1)} \Omega^{(1)}, \\
& \ldots, \text { etc. }
\end{aligned}
$$

Each of these equations is of the same form, the right hand side is known in terms of the solution of the previous equations. The solution of the first order equation is therefore typical. From this one equation we must determine two unknowns, the stochastic $F^{(1)}(t)$ and the nonstochastic $\Omega^{(1)}(t)$. What we do is to determine $\Omega^{(1)}(t)$ as a condition of existence of the solution $F^{(1)}(t)$ satisfying (19). Clearly, forming the mean of this equation, we must have

$$
\Omega^{(1)}(t)=\left\langle K^{(1)}(t)\right\rangle
$$

With this we can solve for $F^{(1)}(t)$ with the initial condition (15). We find

$$
F^{(1)}(t)=\int_{0}^{t} \mathrm{~d} t_{1}\left[K^{(1)}(t)-\left\langle K^{(1)}(t)\right\rangle\right]
$$

In fact, on account of (5) we have $\left\langle K^{(n)}(t)\right\rangle=0$, which means in particular $\Omega^{(1)}(t)$ vanishes. Using this and (23) in the second order equation (21) we find

$$
\Omega^{(2)}\left(t_{1}\right)=\int_{0}^{t_{1}} \mathrm{~d} t_{2}\left\langle K^{(1)}\left(t_{1}\right) K^{(1)}\left(t_{2}\right)\right\rangle
$$

and

$$
F^{(2)}(t)=\int_{0}^{t} \mathrm{~d} t_{1} K^{(2)}\left(t_{1}\right)+\int_{0}^{t} \mathrm{~d} t_{1} \int_{0}^{t_{1}} \mathrm{~d} t_{2}\left[K^{(1)}\left(t_{1}\right) K^{(1)}\left(t_{2}\right)-\left\langle K^{(1)}\left(t_{1}\right) K^{(1)}\left(t_{2}\right)\right\rangle\right] .
$$

In this way we can proceed, successively calculating $F^{(n)}(t)$ and $\Omega^{(n)}(t)$ to any given order.

As $I$ indicated above, the physical observables are expressed in terms of the after-effect function, which satisfies the equation (2) with the successive $\Omega^{(n)}(t)$ now known. Explicitly, using the above results we obtain

$$
\varepsilon \Omega^{(1)}(t)=0, \quad \varepsilon^{2} \Omega^{(2)}(t)=k T \boldsymbol{\sigma} \cdot \mathbf{F}^{-1}[\mathbf{I}-\exp \{-\mathbf{B} t\}] \cdot \boldsymbol{\sigma},
$$

and similar, though increasingly complicated, results in higher order. Here I should remark that the 
cumulant method of van Kampen and Fox yields a result corresponding to the time-ordered exponential solution of eq. (20). But we have not yet finished. The right hand side of (20) is small for small $\varepsilon$, but has a rapid time dependence on the scale of the frictional decay time. This is again a situation, now in a nonstochastic equation, to which the averaging method applies. The solution $\langle R(t)\rangle$ will consist of a slowly varying part, which we denote by $\mathscr{R}(t)$, about which there will be a small amplitude, rapid variation. We accordingly put

$$
\langle R(t)\rangle=\left[1+\varepsilon A^{(1)}(t)+\varepsilon^{2} A^{(2)}(t)+\cdots\right] \mathscr{R},
$$

where $\mathscr{R}$, since it is to exhibit only the slow motion, satisfies an equation of the form

$$
\mathrm{d} \mathscr{R} / \mathrm{d} t=\left[\varepsilon G^{(1)}+\varepsilon^{2} G^{(2)}+\cdots\right] \mathscr{R}
$$

in which the $G$ 's are time-independent operators. As above, putting (27) in (20) and using (28) we get a sequence of equations for determining the $A$ 's and $G$ 's:

$$
\begin{aligned}
& G^{(1)}+\mathrm{d} A^{(1)} / \mathrm{d} t=\Omega^{(1)}(t), \\
& G^{(2)}+\mathrm{d} A^{(2)} / \mathrm{d} t=\Omega^{(2)}(t)+\Omega^{(1)}(t) A^{(1)}(t)-A^{(1)}(t) G^{(1)}, \\
& \ldots, \text { etc. }
\end{aligned}
$$

Again as above, each of these equations is of the same form with the right hand side known in terms of the solutions of the earlier equations. In each equation there are two unknowns, $G^{(n)}$ and $A^{(n)}(t)$, and $G^{(n)}$ must be determined as a condition of existence of a solution $A^{(n)}(t)$ which for long times remains bounded. From (26) we see that in our case the rapid time dependence is an exponential decay, so it is clear that $G^{(n)}$ must be chosen to be the value of the right hand side at $t=\infty$. The solution of the first order equations is therefore

$$
G^{(1)}=\Omega^{(1)}(\infty), \quad A^{(1)}(t)=\int_{0}^{t} \mathrm{~d} t_{1}\left[\Omega^{(1)}\left(t_{1}\right)-\Omega^{(1)}(\infty)\right]
$$

where the constant of integration has been chosen so that

$$
A^{(n)}(0)=0
$$

Continuing, the solution of the second order equation is

$$
G^{(2)}=\Omega^{(2)}(\infty)+\int_{0}^{\infty} \mathrm{d} t\left[\Omega^{(1)}(\infty), \Omega^{(1)}(t)\right]
$$


and

$$
\begin{aligned}
A^{(2)}(t)= & \int_{0}^{t} \mathrm{~d} t_{1}\left\{\Omega^{(2)}\left(t_{1}\right)-\Omega^{(2)}(\infty)\right\} \\
& +\int_{0}^{t} \mathrm{~d} t_{1} \int_{0}^{t_{1}} \mathrm{~d} t_{2}\left\{\Omega^{(1)}\left(t_{1}\right)-\Omega^{(1)}(\infty)\right\}\left\{\Omega^{(1)}\left(t_{2}\right)-\Omega^{(1)}(\infty)\right\}-\int_{0}^{t} \mathrm{~d} t_{1} \int_{t_{1}}^{\infty} \mathrm{d} t_{2}\left[\Omega^{(1)}(\infty), \Omega^{(1)}\left(t_{2}\right)\right] .
\end{aligned}
$$

Here $[A, B] \equiv A B-B A$ is the commutator. Using (26) we see that the first order terms vanish, and the second order terms take the explicit form:

$$
G^{(1)}=k T \boldsymbol{\sigma} \cdot \mathbf{F}^{-1} \cdot \boldsymbol{\sigma}
$$

and

$$
A^{(2)}(t)=-k T \boldsymbol{\sigma} \cdot(\mathbf{F} \cdot \mathbf{B})^{-1} \cdot[\mathbf{1}-\exp \{-\mathbf{B} t\}] \cdot \boldsymbol{\sigma}
$$

In this way we can continue, calculating the terms to a given order, although the calculations become rapidly more laborious as the order increases. The calculations in higher order can be systematized and made somewhat simpler by introducing graphical methods, but I won't go into those here [9].

The integration of (28) is now trivial, since the $G$ 's are time independent. We can therefore express the after-effect function in the explicit form:

$$
\langle R(t)\rangle=\left\{1+\varepsilon A^{(1)}(t)+\varepsilon^{2} A^{(2)}(t)+\cdots\right\} \exp \{G t\}
$$

where

$$
G=\varepsilon G^{(1)}+\varepsilon^{2} G^{(2)}+\cdots
$$

In this form one sees that we have carried the discussion of the after-effect function beyond its expansion as a time-ordered exponential, which would be the result of directly integrating (20), and separated the rapid, short time, behavior, described by the $A$ 's, from the slow long time behavior, described by the exponential. With this I hope I have given some idea of the calculational power of these averaging methods.

\section{References}

[1] G.W. Ford, J.T. Lewis and J. McConnell, Rotational Brownian Motion of an Asymmetric Top, Phys. Rev. A19 (1979) $907-919$

[2] N.G. van Kampen, Stochastic Differential Equations, Phys. Reports 24C (1976) 172-228.

[3] R.F. Fox, Gaussian Stochastic Processes in Physics, Phys. Reports 48 (1978) 179-283.

[4] N.N. Bogoliubov and Y.A. Mitropolsky, Asymptotic Methods in the Theory of Non-Linear Oscillations (Gordon and Breach, 1961).

[5] K.M. Case, A General Perturbation Method for Quantum Mechanics Problems, Prog. Theor. Phys. Supp. 37 (1966) 1-20.

[6] H. Goldstein, Classical Mechanics (Addison-Wesley, 1950).

[7] L.D. Landau and E.M. Lifshitz, Statistical Physics (Pergamon Press, 1958) esp. \$118.

[8] K.M. Case, On Fluctuation-Dissipation Theorems, Trans. Theory and Stat. Phys. 2 (1972) 129-176.

[9] G.W. Ford, J.T. Lewis and J. McConnell, Graphical Study of Rotational Brownian Motion, Proc. Roy. Irish Acad. 76 (1976) $117-143$. 